\title{
Airway resistance measured by the interrupter technique: expiration or inspiration, mean or median?
}

\author{
P.D. Bridge, S.A. McKenzie
}

\begin{abstract}
Airway resistance measured by the interrupter technique: expiration or inspiration, mean or median? P.D. Bridge, S. A. McKenzie. (C) ERS Journals Ltd 2001.

ABSTRACT: The measurement of airway resistance by the interrupter technique (Rint) needs standardization. Should measurements be made be during the expiratory or inspiratory phase of tidal breathing? In reported studies, the measurement of Rint has been calculated as the median or mean of a small number of values, is there an important difference?

Subjects were 2.5 -5.0 yrs (median 4.0 yrs) who had previous respiratory symptoms. The Rint in expiration (RintE) and inspiration (RintI) pre and postsalbutamol, the coefficient of variation (CV) of values contributing to measurements, and bronchodilator responsiveness (BDR) in both phases were compared. Measurements using median and mean were compared.

RintE was higher than RintI by $4 \%(p<0.01)$. The $C V$ of values making up $R$ intE and RintI, and BDR measured in expiration and inspiration were similar. The median difference between means and medians of values making up measurements was $0.6 \%$ (range $-6-11 \%)$.

Rint $\mathrm{E}$ has been shown to be consistently greater then RintI but the difference in this study is small. It is suggested that one or the other is chosen as the standard. In the present data the mean of a set of values contributing to a measurement was not significantly different from the median. However, the use of the median has been recommended since it is less affected by possible outlying values such as might be included by fully automated equipment.
\end{abstract}

Eur Respir J 2001; 17: 495-498.

Although it has been shown that the measurement of airway resistance by the interrupter technique (Rint) is feasible in most children $2-5$ yrs [1] there are no standards for the technique. This makes comparison of Rint studies difficult. Certain aspects of measuring Rint have become accepted, such as supporting the cheeks and pharynx to minimize upper airway compliance [2] and criteria for acceptable mouth-pressure versus time $(P \operatorname{mo}(t))$ transients [3]. Standards for other aspects of Rint measurement need to be agreed upon [4].

Early Rint investigations were inconsistent in the use of the expiratory or inspiratory phase of tidal breathing. More recently, PHAGoO [3], in his work on the analysis of $P \operatorname{mo}(t)$ transients, on which some commercial devices' algorithms are based, used the expiratory phase of tidal breathing to try to obtain a signal with minimum interference from muscular activity [3]. However, others have used inspiration $[5,6]$ because of concern about variations in glottic opening which are more likely to have an effect on the measurement during expiration [7]. Some workers have observed that, during expiration, children sometimes blow in anticipation of the trigger. If there are more technical or physiological difficulties when measuring Rint in expiration (RintE) or inspiration (RintI) these may be reflected in a greater number of unacceptable $P \operatorname{mo}(t)$ transients and may be reflected in a larger coefficient of variation $(\mathrm{CV})$ of the constituent values.
Typically $5-10$ Rint values contribute to a measurement, in studies in young children. It has been suggested that the median of these values should be the measurement because outlying values will affect the average. Most published studies have used the mean $[1,3,5,6]$ and so it is important to know how mean and median compare.

The purpose of this study was to measure the difference between RintE and RintI and to compare the mean and median measurements.

\section{Methods}

\section{Interrupter resistance measurements}

Interrupter resistance was measured using a single commercial device (Microlab 4000; Micro Medical Ltd, Gillingham, UK) throughout the study [1]. Subjects were seated in an identical, comfortable position. They breathed quietly through a cardboard mouthpiece (2.7 $\mathrm{cm}$ diameter or, for some of the younger children, $2.0 \mathrm{~cm}$ diameter) with the nose clipped, the cheeks and pharynx supported and the neck slightly extended. After a period of quiet breathing, in response to a trigger during expiration or inspiration at peak tidal flow, a single shutter closed automatically in $10 \mathrm{~ms}$ and stayed closed for $100 \mathrm{~ms}$. RintE and RintI were 
measured in random order. A switch on the interrupter head allows reversal of polarity of the pressure and, hence, the flow signal allowing measurements to be made in expiration or inspiration. Values were considered acceptable when the $P \operatorname{mo}(t)$ was of consistent shape $[3,8]$. At least six acceptable values of Rint were obtained and the measurement using both the mean and median calculated in a selection of a set of values. The mean of the corresponding flow values was also calculated for each measurement of Rint, before and after bronchodilator. One or two practise attempts were made before the data was recorded. Subjects were unable to anticipate the trigger but were able to hear the shutter closing. Attempts were not accepted if breathing was irregular or the child was restless.

The subject came off the mouthpiece for 3-5 breaths between values and for $30 \mathrm{~s}$ between RintE and RintI. Measurements were repeated 15 min after inhalation of $400 \mu \mathrm{g}$ salbutamol via a spacer device. RintE and RintI were obtained with the values double blind to the technician and patient until completion of the test. For baseline measurements, the total number of interruptions was recorded, in each mode, so that the number of interruptions for six acceptable values could be counted. The time to obtain the baseline measurement, six acceptable values, was recorded to the nearest onequarter of a minute, as this was the shortest time which could be accurately measured.

\section{Subjects}

Subjects included 40 pre-school children, median (range) $4.0(2.5-5.0)$ yrs, with reported respiratory symptoms but were asymptomatic at the time of testing.

\section{Statistical analyses}

To compare RintE with RintI, the data analysed were the means of 5-10 acceptable values (usually 6), as described earlier. These were transformed $\left(\log _{10}\right)$ for analysis [9]. RintE and RintI were compared by expressing the ratio RintE:RintI as were the corresponding mean flows in expiration and inspiration. Bronchodilator responsiveness was expressed as baseline: postsalbutamol ratios. The $\mathrm{CV}(\mathrm{sD} /$ mean $\times 100)$ was calculated for expiratory and inspiratory measurements. Mean and median values for a random selection of 100 sets of values contributing to a measurement were expressed as ratios. This number of means and medians (25 patients, four measurements each) was considered to be enough to make a meaningful comparison.

\section{Results}

Measurements of RintE and RintI are shown in figure 1. The geometric mean ratio RintE:RintI was 1.04 (95\% confidence interval (CI) $1.01-1.07 ; \mathrm{p}=0.018$ ) for baseline measurements and 1.05 (95\% CI 1.02-1.09; $\mathrm{p}<0.01)$ for postsalbutamol measurements. Taking baseline and postsalbutamol measurements together $(n=80)$, the ratio RintE: RintI was inversely related to the average corresponding RintE and RintI measurements (regression coefficient $-0.08, \mathrm{SEM}=0.04, \mathrm{p}=0.03$ ). This means that for every $1.0 \mathrm{kPa} \cdot \mathrm{L} \cdot \mathrm{s}^{-1}$ increase in Rint there is an $8 \%$ decrease in ratio.

Baseline measurements of flow during expiration and inspiration are shown in figure 2. The geometric mean ratio of the flows in expiration and in inspiration was $0.73(95 \%$ CI $0.67-0.80 ; p<0.001)$ for baseline measurements and 0.79 (95\% CI $0.73-0.85$; $\mathrm{p}<0.001)$ for postsalbutamol measurements. There was no correlation between $\log _{10}$ flow measurements at baseline and corresponding $\log _{10}$ Rint measurements (correlation coefficient $-0.0329 ; \mathrm{p}=0.84$ ) and no correlation between the change in flow and corresponding change in Rint between E and I (correlation coefficient= $-0.0716 ; \mathrm{p}=0.66$ ).

There was no difference in the measurement of bronchodilator responsiveness (BDR), the ratio of Rint before bronchodilator to Rint after bronchodilator, in the expiratory or inspiratory phase (RintEbaseline: RintEpostsalbutamol = 1.27; RintIbaseline:RintIpostsalbutamol $=$ 1.31, $\mathrm{p}=0.45)$.

The CV of RintE measurements did not differ from RintI measurements (mean baseline CV E $=15.5 \%$ and $\mathrm{I}=16.5 \%, 95 \%$ CI for difference $=-3.5-1.4$; mean postsalbutamol CV RintE $18.1 \%$ and RintI $18.2 \%$, $95 \% \mathrm{CI}=-3.0-2.9 \%$ ).

The number of interruptions required for six acceptable values did not differ in the expiratory and inspiratory phases (median 6 for both phases; range $6-10)$. There was no difference in the time taken to obtain a set of six values (median $4.5 \mathrm{~min}$; range 4-6.75 $\mathrm{min}$ ).

The mean:median ratios for a random selection of 100 sets of values of $6-9$ per set (each subject had two baseline and two postsalbutamol measurements made)

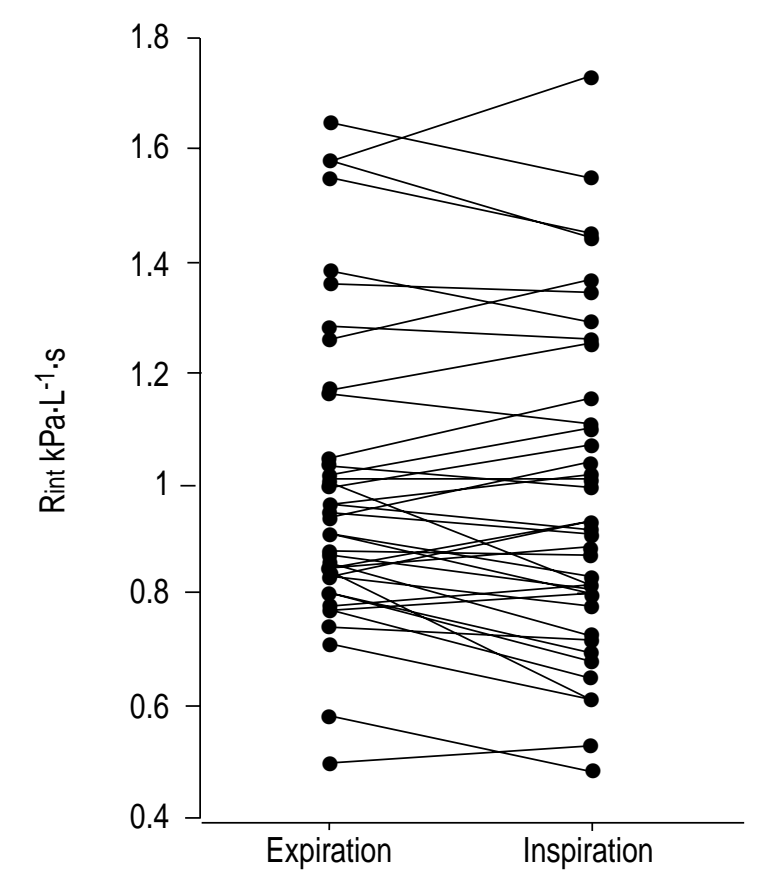

Fig. 1. - Baseline Rint in expiration and inspiration. 


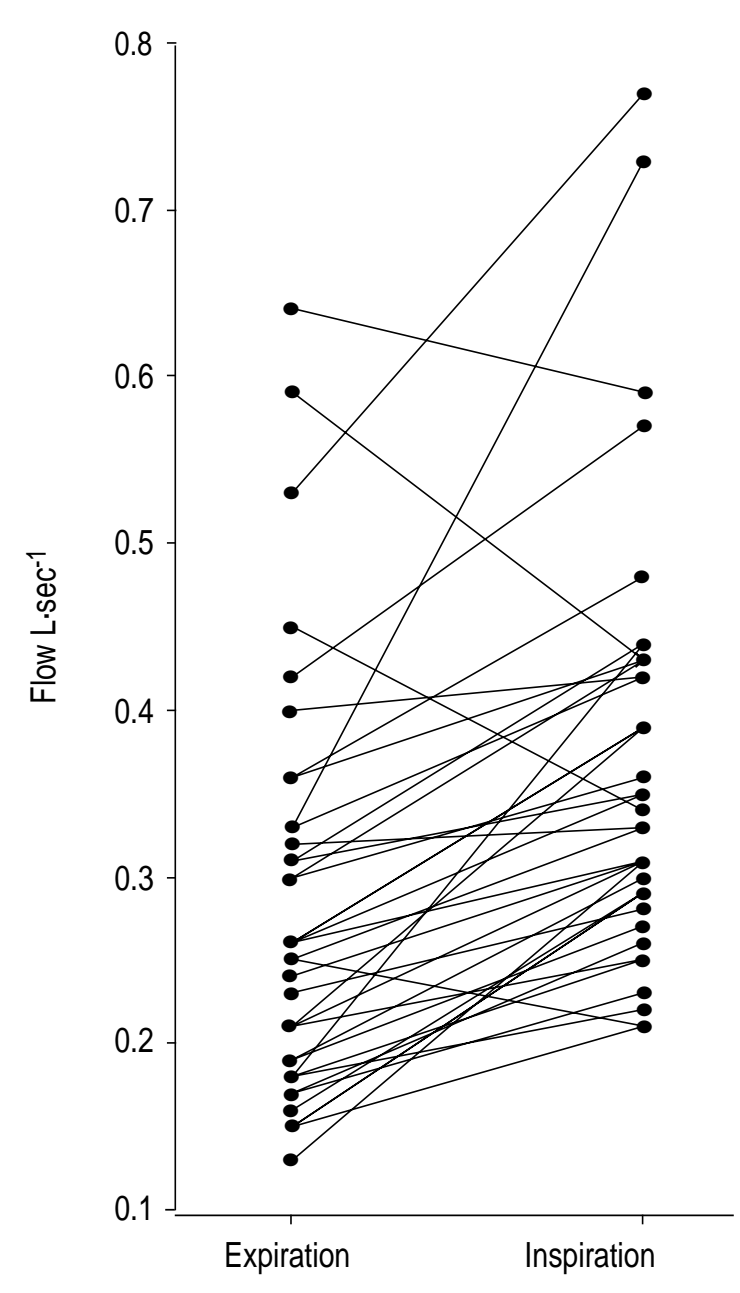

Fig. 2. - Baseline flows in expiration and inspiration.

were not normally distributed. The median ratio was 1.006 (range $0.94-1.11$ ). This implies that for the present data the mean was $-6 \%-11 \%$ from the median.

\section{Discussion}

In this study, a geometric mean difference of $4 \%$ between Rint measurements in the expiratory and inspiratory phases of tidal breathing has been demonstrated. There are slightly higher differences at the lowest measurements made. The measurements in this group of preschool children are similar to those published [10]. Although flows were significantly lower in expiration than in inspiration, no correlation between flow and corresponding Rint measurements and no correlation between change in flow and change in Rint was shown.

It is possible that in airways where there is laminar flow, lateral negative pressure on the airway wall may promote airway narrowing. This, the Bernoulli effect [11] would be expected both in inspiration and in expiration. Another reason for airway narrowing in expiration may be increased airway compliance as intrapleural pressure becomes less negative. Resistances throughout expiration have been demonstrated to be higher than in inspiration in a younger group of recurrently wheezy subjects [12]. The subjects in the present study included children who had previously been wheezy. It is not known whether there is change in glottic diameter during tidal breathing in children, but, if anything, narrowing would be expected in inspiration rather than in expiration due to apposition of supraglottic soft tissues.

Blowing or sucking in anticipation of the trigger in expiration or inspiration was not observed. The device used has a random trigger that occludes after varying numbers of breathing cycles. This should overcome this potential problem.

The system, as currently configured, does not allow for the determination of where interruption occurs in relation to volume. Measurements in the laboratory in similar subjects using a newer system (MicroRint, Micro Medical) which displays flow against time, indicate that interruption occurs at the start of expiration and at the start of inspiration. If these timings correspond to high and low lung volumes respectively, then RintE would be expected to be lower than RintI, the opposite of the present findings. Differences in lung volume at the time of interruption in the two phases would not, then, explain the differences in resistance.

The repeatability of the measurement in the laboratory, ( $2 \mathrm{SD}$ of the mean ratio of two measurements $30 \mathrm{~s}$ apart) [1] is $\pm 16 \%$. The difference between measurements in expiration and inspiration is small by comparison. Only two other studies, in older children, have systematically investigated RintE and RintI [2,8] and have shown RintE to be greater than RintI. Differences are of the order of $20 \%$ in both, much higher than has been demonstrated. There is no explanation for this other than noting that the age and health status of subjects, and methods are different.

There are various methods used to describe BDR. The ratio of baseline to postbronchodilator was chosen. No difference in BDR measured in the expiratory or inspiratory phases was found, as others have shown [8].

A within-subject CV of the set of values making up a measurement has been considered to be acceptable if it is $<20 \%$ [2]. The CV of the values is on average $<20 \%$. $\mathrm{CV}$ measured in expiration or in inspiration is similar. There was no difference between expiration and inspiration in the number of interruptions needed to obtain acceptable values, nor the time to do the test. It is recommended that the median of the six or so values should be used as the measurement, as this is less affected than the mean by outlying values. Such values cannot be avoided with fully automated equipment in which transients cannot easily be examined for acceptability. Some commercial devices collect values which are considered by the machine's software to be acceptable. Inexperienced operators may overlook outlying values which could have resulted from poor technique, such as moving the head or blowing the cheeks out. It has been shown that there was very little difference between mean and median values in the data, so that studies which have used the mean are still valid $[1,3,5,6]$.

In conclusion, this study has shown that interrupter resistance in expiration is $4 \%$ higher than in inspiration, 
a value much smaller than the repeatability of the measurement. The mean and median of values which contribute to a measurement using this method differ very little. The authors agree with CARTER [4] that there is a need for standardization of all aspects of interrupter resistance measurement.

\section{References}

1. Bridge PD, Ranganathan S, McKenzie SA. The measurement of airway resistance using the interrupter technique (Rint) in pre-school children in the ambulatory setting. Eur Respir J 1999; 13: $792-796$.

2. Oswald-Mammosser M, Llerena C, Speich JP, Donata $\mathrm{L}$, Lonsdorfer L. Measurements of respiratory system resistance by the interrupter technique in healthy and asthmatic children. Pediatr Pulmonol 1997; 24: 78 -85.

3. Phagoo SB, Wilson NM, Silverman M. Evaluation of a new interrupter device for measuring bronchial responsiveness and the response to bronchodilator in 3 year old children. Eur Respir J 1996; 9: 1374-1380.

4. Carter ER. It is time to consider standardizing the interrupter technique. Eur Respir J 1997; 10: $1428-$ 1429.

5. Bisgaard H, Klug B. Lung function measurement in awake young children. Eur Respir J 1995; 8: 2067-2075.

6. Klug B, Bisgaard H. Measurement of lung function in awake 2-4-year-old asthmatic children during methacholine challenge and acute asthma: a comparison of the impulse oscillation technique, the interrupter technique, and transcutaneous measurement of oxygen versus whole-body plethysmography. Pediatr Pulmonol 1996; 21: 290-300.

7. Stanescu DC, Clement J, Pattijn J, Woestijne KP. Glottis opening and airway resistance. $J$ Appl Physiol 1972; 32: $460-466$.

8. Carter ER, Stecenko AA, Pollock BH, Jaeger MJ. Evaluation of the interrupter technique for the use of assessing airway obstruction in children. Pediatr Pulmonol 1994; 17: $211-217$.

9. Healy MJR. The disciplining of medical data. Brit Med Bull 1968; 24: 210-214.

10. Klug B, Bisgaard H. Specific airway resistance, interrupter resistance, and respiratory impedance in healthy children aged 2-7 years. Pediatr Pulmonol 1998; 25: $322-331$.

11. Bates JHT, Turner MJ, Lanteri CJ, Jonson B, Sly PD. Measurement of Flow and Volume. In: Stocks J, Sly PD, Tepper RS, Morgan WJ, eds. Infant Respiratory Function Testing. New York, John Wiley and Sons, Inc Publication, 1996, pp. 81-109.

12. Springer C, Vilozni D, Bar-Yishay E, Avital A, Noviski N, Godfrey S. Comparison of airway resistance and total respiratory system resistance in infants. Am Rev Respir Dis 1993; 148: 1008 - 1012. 